\title{
THE SPIN-ORBIT SPLITTING IN THE Si BANDSTRUCTURE MEASURED BY MEANS OF SPIN-RESOLVED PHOTOEMISSION
}

\author{
B. Schmiedeskamp, B. Vogt and U. Heinzmann \\ Fakultät für Physik, Universität Bielefeld, D-4800 Bielefeld, Germany \\ and \\ Fritz-Haber-Institut der Max-Planck-Gesellschaft, D-1000 Berlin, Germany
}

(Received 30 April 1990 by G. Güntherodt)

\begin{abstract}
Spin-, angle- and energy-resolved photoemission with circularly polarized synchrotron radiation from BESSY has been applied to deduce information about the electronic structure of silicon in the $\Lambda$-direction. We used the highly symmetrical experimental set-up of normal radiation incidence and normal electron emission. This method, which has in the past several times been applied to metals and rare gas crystals with medium and high atomic numbers, has thus been applied now to a semiconductor with a low atomic number $(Z=14)$. For photon energies between 5.5 and $10 \mathrm{eV}$ we find a peak $V$ in the photoemission spectra which corresponds to a transition from the valence bands in the bulk band structure of silicon in the $\Lambda$-direction. The electrons which contribute to the peak are spin polarized due to a spin-orbit splitting $\Delta E_{\mathrm{so}}$ smaller than $50 \mathrm{meV}$. Adsorption of $0.5 \mathrm{ML} \mathrm{Ag}$ or $\mathrm{Au}$ induces band bendings and work function changes and yields a sharper peak and less secondary electron background than the clean $\mathrm{Si}\left(\begin{array}{lll}1 & 1 & 1\end{array}\right)$-surface. For both the clean and adsorbate covered surface and $k_{1}$ away from $\Gamma$ we determine $\Delta E_{\text {so }}$ from a spin analysis of peak $V$ and find values between 20 and $35 \mathrm{meV}$ with typical errors of $10 \mathrm{meV}$. These values in $\Lambda$-direction are smaller than the $\Delta E_{\mathrm{so}}$-value of $44 \mathrm{meV}$ at the $\Gamma$-point [1, 2].
\end{abstract}

\section{INTRODUCTION}

ANGLE- AND energy-resolved photoemission is an often used method for bulk bandstructure determination [3-7] and references therein. In the past the method has mainly been applied to metals while fewer studies were performed on semiconductors [3-7]. Examinations of the bulk bandstructure of $\mathrm{Si}$ in the $\Lambda$-direction were performed by Uhrberg $e t$ al. [8]. For photon energies between 10 and $17 \mathrm{eV}$ they demonstrate that transitions exist from the valence bands into a free electron like final band.

Spin-resolved photoemission with circularly polarized radiation from non magnetic crystals yields further information about the electronic bandstructure, especially with respect to the symmetry of bands involved in transitions, spin-orbit splittings and hybridization [5-7, 9-14]. With spin-resolved and energy-integrated photoemission Bona et al. [1] show that a spin-orbit splitting smaller than $50 \mathrm{meV}$ exists at the $\Gamma$-point of the silicon bulk bandstructure.

In this work we study the bulk bandstructure of silicon in the $\Lambda$-direction with spin-, energy- and angle-resolved photoemission. For photon energies between 6 and $10 \mathrm{eV}$ we study transitions from the valence bands into the first final band above the vacuum level and determine the valence band spinorbit splittings for several $K_{\perp}$-values away from $\Gamma$. The same procedure is applied to Si( 111$)$ surfaces which were covered with 0.5 monolayers $\mathrm{Ag}$ or $\mathrm{Au}$.

\section{EXPERIMENTAL}

The experiments were performed with circularly polarized radiation from the storage ring BESSY in an apparatus which is described in [7]. We used the highly symmetrical experimental set-up of normal radiation incidence and normal electron emission. Angle- and energy-analysis of the photoelectrons was accomplished with an electron spectrometer which is described in [15]. The overall energetic resolution (electrons plus photons) was better than $150 \mathrm{meV}$, the angular resolution $\pm 3^{\circ}$. The $\operatorname{Si}\left(\begin{array}{lll}1 & 1 & 1\end{array}\right)$-sample was an $n$-type $(10-20 \Omega \mathrm{cm})$ single crystal which was aligned within 
0.5 degrees with the [lllll-direction and within $0.3^{\circ}$ with the light direction. The samples were heated to $900^{\circ} \mathrm{C}$ at background pressures in the $10^{-10} \mathrm{mbar}$ range in order to remove an oxide protection layer and to prepare an ordered $(7 \times 7)$ silicon surface. The base pressure in the system was lower than $3 \times$ $10^{-10}$ mbar. The crystal surface has been controlled by Auger electron spectroscopy (AES) and LEED. The metal coverages were obtained by evaporating 0.5 monolayers of $\mathrm{Ag}$ or $\mathrm{Au}$, while the sample was kept at room temperature. We used resistively heated evaporators which are surrounded by a cooled shield [16]. The metal beams pass, in addition, a $1 \mathrm{~cm}$ diameter tube before they reach the silicon surface which is positioned at a distance of $20 \mathrm{~cm}$ from the source. After evaporation the metal covered Si-crystal was heated to $500^{\circ} \mathrm{C}$ and revealed then a clear $\sqrt{ } 3 \times \sqrt{ } 3 R 30^{\circ}$ structure for $\mathrm{Ag}$ (denoted as $\operatorname{Si}\left(\begin{array}{lll}1 & 1 & 1\end{array}\right)-\sqrt{ } 3 \mathrm{Ag}$ ) and a $5 \times 1$ structure for $\mathrm{Au}$ (denoted as $\operatorname{Si}(111)(5 \times 1) \mathrm{Au}$ ).

\section{RESULTS AND DISCUSSION}

Photoemission spectra obtained for the clean silicon surface are given in Fig. 1. The left column shows non-spin-resolved data for photon energies between 5 and $10 \mathrm{eV}$. One peak $(V)$ is resolved in the spectra. It emerges out of the secondary electron background at $h v \approx 7 \mathrm{eV}$, is best resolved at $8 \mathrm{eV}$ and decreases then rapidly for higher photon energies. The binding energy of peak $V$ increases continuously with photon energy. The corresponding spin-resolved photoemission spectra are given in the right part of Fig. 1. Circles are the non-spin-resolved photoemission intensities $I$. By means of the measured spinpolarization $P$ they are separated into the partial intensities $I_{+}=I / 2(1+P)$ and $I_{-}=I / 2(1-P) . I_{+}$ and $I_{\text {. }}$ (the dashed and solid lines in the right column of Fig. 1) denote then the electron intensities with spin parallel and antiparallel to the photon spin, respectively. It is obvious from the data that the electrons which contribute to peak $V$ in the spectra in the right column of Fig. 1 are slightly spin-polarized with the $I$-part dominating at lower binding energies. For an interpretation of the data we regard the bulk bandstructure of silicon in the $\Lambda$-direction which is given in Fig. 2. The bandstructure, which is a calculation of Persson, is taken from [8].

The slight dispersion of peak $V$ in Fig. 1 and its drastic intensity decrease at $h v \approx 9 \mathrm{eV}$ fit excellently into the picture that peak $V$ is due to transitions from the $\Lambda_{3}$-valence band in Fig. 2 into the first calculated $\Lambda_{1}$-final band above the vacuum level. This is indicated by the arrows in Fig. 2 . The bandstructure in Fig. 2 is a non-relativistic calculation. From relativistic

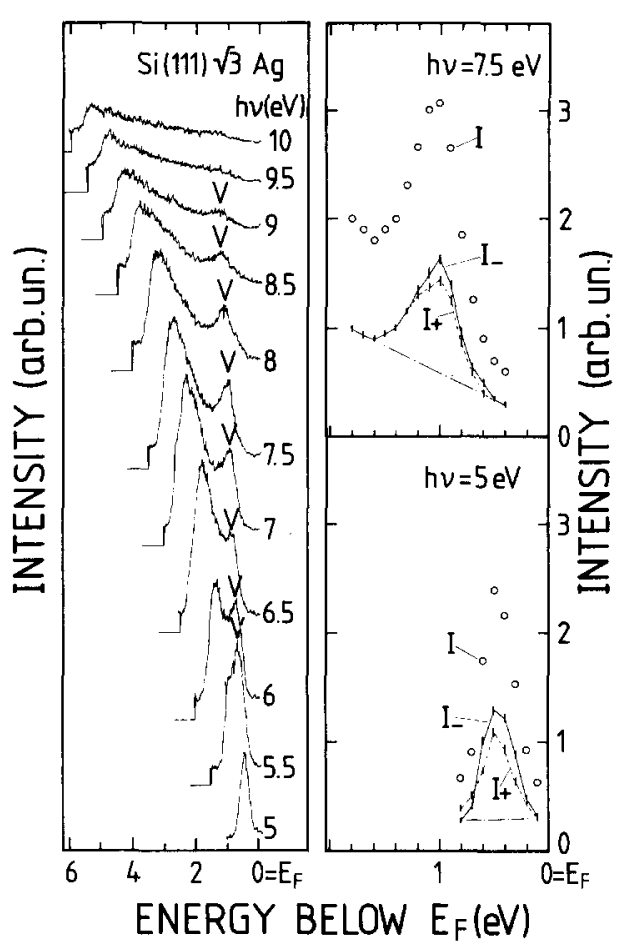

Fig. 1. Si( $\left.\begin{array}{lll}1 & 1 & 1\end{array}\right)$ photoemission spectra. Left: non-spinresolved spectra for different photon energies. $V$ indicates a peak, which corresponds to a direct transition in the Si bulk bandstructure. Right: spinresolved photoemission spectra for 3 photon energies. Circles represent the total intensity $I$. The dashed and solid lines correspond to the $I_{+}$and $I_{-}$intensities, respectively. The dashed dotted line indicates the secondary electron background used in the data analysis for the determination of the spin-orbit splittings $\Delta E_{\mathrm{so}} . \Delta E_{\mathrm{so}}$ was obtained from the centers of gravity of the background corrected $I_{+}$- and $I_{-}$-peak intensities. The vertical bars in the $I_{+}$- and $I_{-}$intensities represent the total experimental error including the statistical error of the count rates and the uncertainties of the light polarization and the detector asymmetry function.

calculations for several diamond lattice crystals [17] it is known that the $\Lambda_{3}$-valence band of the nonrelativistic bandstructure splits into a $\Lambda_{4,5}^{3}$-band and a $\Lambda_{6}^{3}$-band due to spin-orbit interaction with the $\Lambda_{4,5}^{3}$-band at lower binding energy. For a transition from a $\Lambda_{4,5}^{3}$-band into a $\Lambda_{6}^{1}$-band we expect negatively spin-polarized photoelectrons [18]. This is indeed what we see in the right column of Fig. 1; it supports thus the opinion that this spin-polarization is due to the spin-orbit splitting of the $\Lambda_{3}$-valence band.

A similar set of data was measured for $\operatorname{Si}\left(\begin{array}{lll}1 & 1 & 1\end{array}\right)$ surfaces covered with 0.5 monolayer $\mathrm{Ag}$ and $\mathrm{Au}-$ adsorbates as described above. Results for the $\mathrm{Si}\left(\begin{array}{lll}1 & 1 & 1\end{array}\right)-$ $\sqrt{3} \mathrm{Ag}$ surface are given in Fig. 3. The left and right 


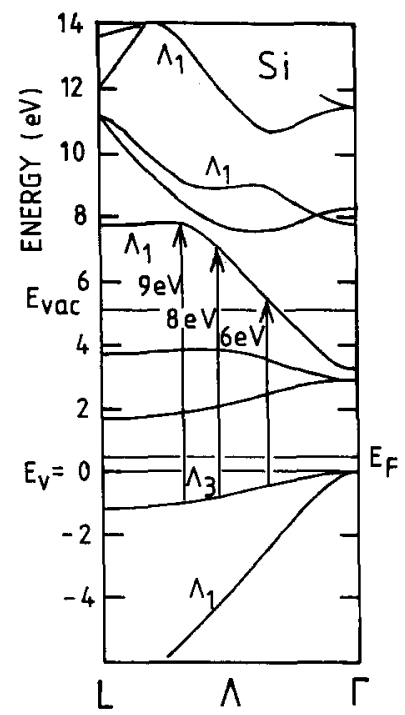

Fig. 2. Bandstructure of silicon in the $\Lambda$-direction (from [8]). The calculations, which are non relativistic, are from Persson. Arrows indicate possible transitions between 6 and $9 \mathrm{eV}$ photon energy.

columns show again the non-spin-resolved and spinresolved spectra. Compared to the spectra in Fig. 1 we find a slightly reduced work function, a bending of the valence bands by about $0.2 \mathrm{eV}$ towards lower binding energy, a lower secondary electron background and a sharper peak $V$ which is probably due to a more perfect ordering of the Si surface in the $\sqrt{ } 3$ structure. These findings allow a study of the spin-polarization behaviour of peak $V$ also for lower photon energies, as can be seen in the right column of Fig. 3 . We get more than 10 percent spin-polarization for electrons with energies just above the vacuum level while the spinresolved photoemission spectrum for $h v=7.5 \mathrm{eV}$ differs slightly (at the high binding energy side of the $I_{+}$and $I_{-}$-peaks) from the $8 \mathrm{eV}$ spectrum in Fig. 1 . Spectra for the Au covered surface (not displayed) agree with respect to the shape of peak $V$ and the secondary electron background with the $\mathrm{Ag}$ covered surface and yield also spin-polarized electrons.

An apparently consistent result is that the integrated $I_{+}$intensity is in all the measured spectra less than that of $I_{-}$. This is possibly due to hybridization of the $\Lambda_{1}$ - and $\Lambda_{3}$-valence band in Fig. 2. As already described above a relativistic bandstructure calculation yields an additional splitting of the $\Lambda_{3}$-band into a $\Lambda_{6}^{3}$ - and a $\Lambda_{45}^{3}$-band, while the $\Lambda_{1}$-band becomes a $\Lambda_{6}^{1}$-band. One expects then also hybridization and an avoided crossing point of the $\Lambda_{6}^{1}$ - and the $\Lambda_{6}^{3}$-band near $\Gamma$ [17]. Earlier spin resolved studies of $\operatorname{Pd}\left(\begin{array}{lll}1 & 1 & 1\end{array}\right)$ [11] yielded indeed lower photoelectron intensities for

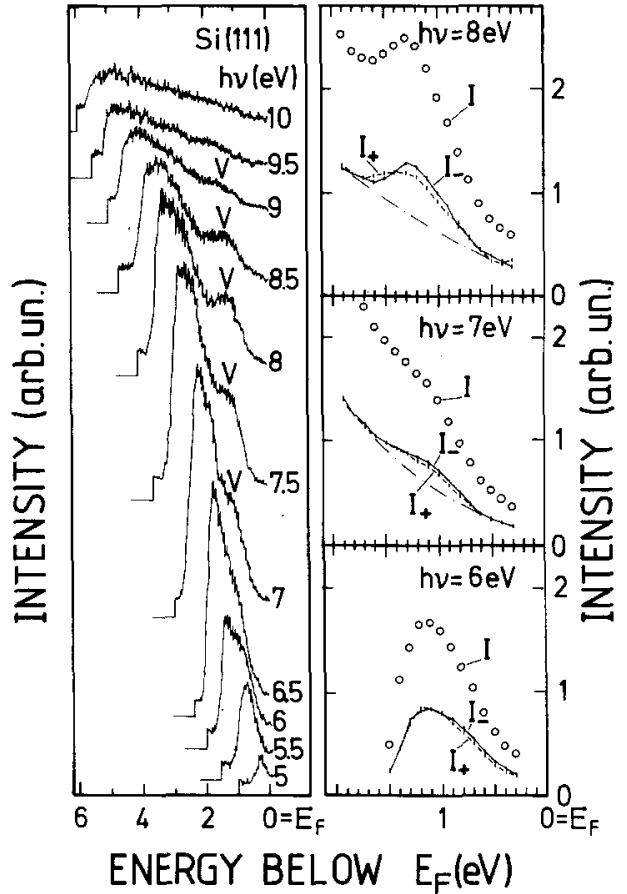

Fig. 3. Spectra for photoemission from a $\operatorname{Si}\left(\begin{array}{lll}1 & 1 & 1\end{array}\right)-\sqrt{ } 3$ $\mathrm{Ag}$ surface. Left: non-spin-resolved spectra. The direct-transition-peak $V$ is sharper than peak $V$ in Fig. 1. Right: spin-resolved photoemission spectra for 5 and $7.5 \mathrm{eV}$ photon energy. Meaning of the symbols as in Fig. 1.

transitions from a $\Lambda_{6}^{3}$-band in the neighbourhood of an avoided crossing point [19] with a $\Lambda_{6}^{1}$-band. This explains especially the high spin polarization values in the $5 \mathrm{eV}$ spectrum in the lower right part of Fig. 3 .

We use both the spin-resolved and the non-spinresolved data of the clean and metal covered $\mathrm{Si}\left(\begin{array}{lll}1 & 1 & 1\end{array}\right)$ surfaces to perform a spin-resolved bandmapping of the $\Lambda_{3}$ valence bands. The upper part of Fig. 4 displays the binding energies of peak $\mathrm{V}$ as determined from the non-spin-resolved spectra in Figs. 1 and 3. A comparison of this binding energies with the bandstructure in Fig. 2 shows that very good agreement is obtained with the calculated $\Lambda_{3}$-band. In the lower part of Fig. 4 we present the values for the spin-orbit splitting $\Delta E_{\mathrm{so}}$ which have been determined from the spinresolved photoemission spectra. Squares are the data for the clean $\mathrm{Si}$-surface, circles and triangles represent the $\Delta E_{\mathrm{so}}$-values for the $\mathrm{Ag}$ - and Au-covered surfaces, respectively. We obtain values between about 20 and $35 \mathrm{meV}$ with typical errors of $10 \mathrm{meV}$ not depending on whether the Si surface is metal covered or not. It is worth noting, that the experimentally obtained spinorbit splitting of about $30 \mathrm{meV}$ is a factor of 4 smaller than the resolution of our electron spectrometer used. 


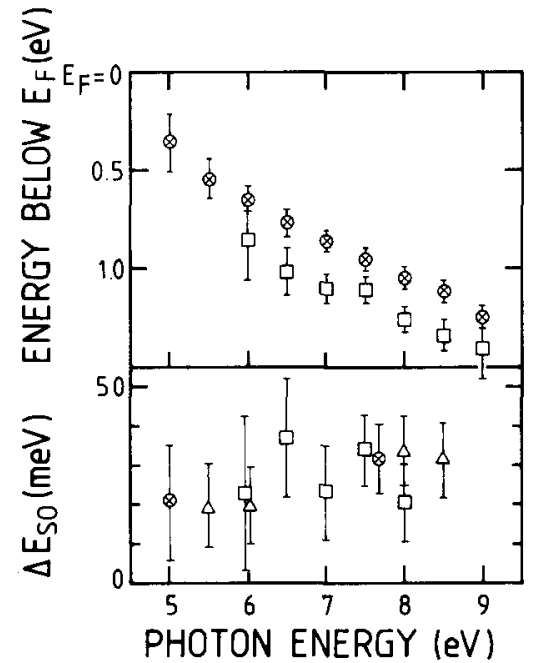

Fig. 4. Energy below $E_{F}$ and spin-orbit splitting $\Delta E_{\text {so }}$ for the direct-transition-peak $V$ vs photon energy. $\square: \mathrm{Si}\left(\begin{array}{lll}1 & 1 & 1\end{array}\right)$-surface, $\otimes: \mathrm{Si}\left(\begin{array}{lll}1 & 1 & 1\end{array}\right)-\sqrt{ } 3 \mathrm{Ag}$ surface, $\Delta: \operatorname{Si}(111)(5 \times 1)$ Au surface.

It demonstrates again [11], that the spin-polarization analysis of photoelectron peaks increases the overall energetic sensitivity compared to non-spin-resolved photoelectron spectroscopy. Finally, we compare our results with the $\Delta E_{\mathrm{SO}}$-value, which is known to be $44 \mathrm{meV}$ at the $\Gamma$-point $[1,2]$ and note that our values which were all obtained for $K_{\perp}$-values away from $\Gamma$ are systematically lower.

\section{CONCLUSIONS}

We have studied the electronic structure of $\mathrm{Si}$ in the $\Lambda$-direction with spin-, angle- and energy-resolved photoemission. One peak $V$ is resolved in the photoemission spectra. $V$ corresponds to transition from a $\Lambda_{3}$-valence band into the first unoccupied band above the vacuum level. The electrons which contribute to $V$ are spin-polarized due to the spin-orbit splitting of the $\Lambda_{3}$-band. Analysis of the data leads to values of the spin-orbit splittings of this band between 20 and $35 \pm 10 \mathrm{meV}$. The same studies were done for well ordered $\mathrm{Si}\left(\begin{array}{lll}1 & 1 & 1\end{array}\right)-\sqrt{ } 3 \mathrm{Ag}$ and $\operatorname{Si}\left(\begin{array}{llll}1 & 1 & 1\end{array}\right)(5 \times 1) \mathrm{Au}$ surfaces. While work functions and band bending are altered by the metal adsorption the spin-orbit splitting of peak $V$ seems to be unaffected by the metal adsorption.
Acknowledgements - We acknowledge discussions with N. Irmer, N. Müller and P. Stoppmanns as well as the support of the BESSY staff. The work was financially supported by the BMFT (05431).

\section{REFERENCES}

1. G.L. Bona \& F. Meier, Solid State Commun. 55, 851 (1985).

2. S. Zwerdling, K.J. Button, B. Lax \& L.M. Roth, Phys. Rev. Lett. 4, 173 (1960).

3. F.J. Himpsel, Adv. Phys. 32, 1 (1983).

4. R. Courths \& S. Hüfner, Phys. Rep. 112, 53 (1984).

5. U. Heinzmann, Phys. Scripta T17, 77 (1987).

6. H.P. Oepen, K. Hünlich, J. Kirschner, A. Eyers, F. Schäfers, G. Schönhense \& U. Heinzmann, Phys. Rev. B31, 6846 (1985).

7. A. Eyers, F. Schäfers, G. Schönhense, U. Heinzmann, H.P. Oepen, K. Hünlich, J. Kirschner \& G. Borstel, Phys. Rev. Lett. 52, 1559 (1984).

8. R.I.G. Uhrberg, G.V. Hansson, U.O. Karlsson, J.M. Nicholls, P.E.S. Persson, S.A. Flodström, R. Engelhardt \& E.E. Koch, Phys. Rev. B31, 3795 (1985).

9. B. Kessler, A. Eyers, K. Horn, N. Müller, B. Schmiedeskamp, G. Schönhense \& U. Heinzmann, Phys. Rev. Lett. 59, 331 (1987).

10. N. Müller, B. Kessler, B. Schmiedeskamp, G. Schönhense \& U. Heinzmann, Solid State Commun. 61, 187 (1987).

11. B. Schmiedeskamp, B. Kessler, N. Müller, G. Schönhense \& U. Heinzmann, Solid State Commun. 65, 665 (1988).

12. J. Garbe, D. Venus, S. Suga, C. Schneider \& J. Kirschner, Surf. Sci. 178, 342 (1986).

13. J. Garbe \& J. Kirschner, Phys. Rev. B39, 1530 (1989).

14. R. Allenspach, F. Meier \& D. Pescia, Phys. Rev. Lett. 51, 2148 (1983).

15. K. Jost, J. Phys. E12, 1001, 1006 (1979).

16. B. Vogt, B. Schmiedeskamp \& U. Heinzmann, to be published.

17. J.R. Chelikowsky \& M.L. Cohen, Phys. Rev. B14, 556 (1976).

18. M. Wöhlecke \& G. Borstel, in Optical Orientation, (Edited by F. Meier \& B.P. Zakharchenya), Elsevier Science Publ. 1984; one should note that we denote the spin-polarization as positive, if electron and photon spin are parallel; this is just opposite to the definition given by Wöhlecke and Borstel.

19. See the spin-resolved photoemission spectra for $9 \mathrm{eV}$ photon energy in Fig. 3 of [11]. 